\title{
Tabaquismo materno como un factor posiblemente implicado en el desarrollo de la obesidad infantil
}

\author{
Juan Sebastián Sanabria C. ${ }^{a}$, Jaime David Arce S. ${ }^{a}$, Orlenis María Sierra O. ${ }^{a}$, Aura \\ María Gil V.b
}

Grupo de Investigaciones Biomédicas Uniremington. Facultad de Ciencias de la Salud. Corporación Universitaria Remington. Medellín, Colombia.

a Médico. ${ }^{\mathrm{b}}$ Bióloga, PhD.

\section{RESUMEN}

El tabaquismo es la adicción al tabaco provocada principalmente por diversos componentes activos y tóxicos como la nicotina. El consumo de cigarrillo durante la gestación puede provocar desprendimiento de placenta, placenta previa, embarazo ectópico y aborto espontáneo, como también inducir alteraciones en el feto. En la presente revisión de la literatura se recopiló información en bases de datos como PubMed, Embase y Google Académico, concerniente a los posibles efectos del tabaquismo materno durante la gestación sobre el desarrollo de la obesidad infantil. Fueron seleccionados 38 artículos escritos en el idioma inglés y español, publicados a partir de año de 1988 hasta el año 2015, que incluyeron metaanálisis, artículos originales, y revisiones de tema. Se encontró que la exposición al humo del tabaco durante la gestación ha sido ampliamente descrita como un factor de riesgo para la manifestación de alteraciones en el desarrollo fetal como retardo del crecimiento intrauterino y bajo peso al nacer. Además, se ha asociado ampliamente con trastornos del desarrollo infantil en etapas avanzadas, como preescolares y escolares, manifestados en un aumento del índice de masa corporal con respecto al percentil adecuado para la edad; incremento de la incidencia de sobrepeso y obesidad en el infante. Se concluye que la exposición al humo del cigarrillo durante la gestación se relaciona con alteraciones en el crecimiento del niño y en el desarrollo de enfermedades prevalentes asociadas a la obesidad.

\section{PALABRAS CLAVES: Tabaquismo pasivo, tabaquismo maternal, obesidad pediátrica, nicotina, represión epigenética}

\section{SUMMARY}

Most smokers use tobacco regularly because they are addicted to various active and toxic compounds such as nicotine. Cigarette smoking during pregnancy can cause abruption, placenta previa, ectopic pregnancy and spontaneous abortion, as well as induce alterations in the fetus. In this review, information was collected in databases such as PubMed, Embase and Google Scholar, concerning the possible effects of maternal smoking during pregnancy on the development of childhood obesity. Thirty-eigth articles written in English and Spanish published from year 1988 to 2015, which included meta-analysis, original articles and reviews were selected topic. It was found that exposure to cigarette smoke during pregnancy has been widely described as a risk factor for alterations in fetal development such as intrauterine growth retardation and low birth weight. In addition, it has been widely associated with disorders of child development in advanced stages, preschool and school age: increased body mass index regarding the appropriate percentile 
for age, and increase in childhood overweight and obesity. It is concluded that exposure to cigarette smoke during pregnancy is associated with changes in child growth and development of prevalent diseases associated with obesity.

KEY WORDS: Tabaquism, maternal tabaquism, pediatric obesity, nicotine, epigenetic repression

\section{INTRODUCCIÓN}

El consumo de tabaco es una de las principales causas de mortalidad prevenible en el mundo, ya que es uno de los principales productos de consumo legal perjudicial para todos los expuestos, causando el $50 \%$ de muertes a sus consumidores (1). Para el año 2010, el 12,3\% de madres en Estados Unidos fumaron durante su embarazo, lo cual ha sido implicado en diversas patologías obstétricas y neonatales asociados principalmente a la nicotina, que puede ocasionar que su concentración en la circulación fetal aumente incluso hasta un 15\% comparado con la concentración en la circulación materna (2-4).

El desarrollo intrauterino, una ventana de tiempo crítica en la génesis de alteraciones del desarrollo humano a través de la teoría de "programación fetal" descrita entre 1980-1990 por David Barker, se presentó como el primer modelo que explica la interacción entre los genes y el entorno intrauterino como factores que influyen en el posterior desarrollo del individuo $(5,6)$. Condiciones intrauterinas adversas pueden influir negativamente sobre la adecuada programación genética fetal, lo cual podría repercutir en la salud del neonato a corto y/o largo plazo, ocasionando posiblemente un mayor riesgo de presentar enfermedades metabólicas y obesidad (4).

El objetivo de la presente revisión de la literatura fue recopilar información sobre el posible efecto del tabaquismo materno sobre el posterior desarrollo de obesidad en el infante.

\section{MATERIALES Y MÉTODOS}

Se realiza una revisión de la literatura en los idiomas español e inglés, utilizando como herramienta las bases de datos PubMed, Embase y Google Académico, con una búsqueda bibliográfica adicional en el programa EndNote 7.2, usando las palabras claves clave: obesidad pediátrica, embarazo, nicotina y represión epigenética. Se incluyó la webgrafía de la Organización Mundial de la Salud (OMS), artículos tipo reporte de caso, revisión de tema, artículos originales y metaanálisis, que incluyeron estudios observacionales y experimentales. De esta manera, se seleccionaron 39 artículos entre los años 1988 hasta 2015, los cuales abordaron las siguientes temáticas: incidencia y prevalencia del tabaquismo materno, tabaquismo durante la gestación, madres fumadoras durante el embarazo con efecto directo sobre el peso del infante; tabaquismo materno y paterno como factor implicado en el desarrollo de la obesidad infantil. La información más relevante fue recopilada en la Tabla I.

\section{Incidencia, prevalencia y epidemiologia del tabaquismo}

El tabaquismo es una de las causas de mortalidad prevenible en el mundo, cuyas manifestaciones clínicas se presentan a largo plazo (1). En el mundo mueren alrededor de 6 millones de personas por consumir cigarrillo. Las principales causas de muerte por consumo de tabaco son el desarrollo de cáncer de pulmón con un $71 \%$, enfermedades respiratorias crónicas $\sim 42 \%$ y en un $10 \%$ por enfermedades cardiovasculares (7).

El tabaquismo junto con el consumo excesivo de alcohol, la mala alimentación y la falta de actividad física, son los principales factores de riesgo para el padecimiento de enfermedades cardiovasculares, cáncer, enfermedades pulmonares crónicas y diabetes, las cuales conllevan al $80 \%$ de las muertes por enfermedades no transmisibles. De acuerdo con estimaciones de la organización mundial de la salud (OMS), las enfermedades no transmisibles contribuyeron con 36 millones de muertes en el mundo en 2008, representando el $63 \%$ de las 57 millones de muertes totales (8), correspondiendo a un $54 \%$ de los años vida ajustado por discapacidad en 2010 a nivel mundial por enfermedades no transmisibles (9). Lo anterior, ha provocado implicaciones económicas enormes, debido a la suma de costos en atención de salud y la baja productividad económica, debido a la enfermedad y a muertes prematuras. Se ha calculado costos de 47 billones de dólares entre 2011 y 2030 a causa de las enfermedades no transmisibles y enfermedades mentales (10). 


\section{Tabla I \\ ESTUDIOS CON RELEVANCIA EPIDEMIOLÓGICA Y BIOQUÍMICA DEL ARTÍCULO}

\begin{tabular}{ll}
\hline REFERENCIA & \multicolumn{1}{c}{ DEFINICION O RESULTADOS } \\
\hline \multicolumn{1}{c}{ INCIDENCIA, PREVALENCIA Y } \\
EPIDEMIOLOGIA
\end{tabular}

CONCLUSIONES Y/O

RECOMENDACIONES

y OR 1,23 respectivamente].

\section{FACTORES BIOQUIMICOS Y MOLECULARES}

Las personas expuestas a un ambiente adverso en el útero, están expuestos a un entorno adverso en la vida adulta y que esto más adelante, el medio ambiente produce los efectos atribuidos a la programación.

Se identificaron con estudios en cobayos, al que estuvieron con restricción nutricional in útero, un aumento en la actividad adenohipofisiaria, conllevando el aumento del cortisol, hormona anabolizante implicada en el anabolismo de los lípidos y glúcidos.

El aumento crónico de la presión sanguínea, que es inducida por los glucocorticoides, se ve reflejado en una reducción de la 11 beta deshidrogenasa de esteroide (11-betaOHSD).

La asociación de pequeño tamaño en el nacimiento con desarrollo de obesidad central, resistencia a la insulina y el síndrome metabólico.

"La teoría de Barker", explica el desarrollo de múltiples enfermedades metabólicas y cardiovasculares en el niño y adulto; basándose en tres fases, la $1^{\circ}$ donde hay una alteración en los factores nutricionales, $2^{\circ}$ fase caracterizada por cambios bioquími$\cos$ y fisiológicos $3^{\circ}$ fase donde se encuentra establecida la patología final.

Los múltiples factores asociados a un mayor riesgo en el desarrollo de sobrepeso y obesidad en la infancia.
El tabaquismo matero durante el embarazo se asoció positivamente con la obesidad y el sobre peso en la población estudiada en Japón a los 5 años y con posterior confirmación en la revaloración a los 9 años.

Independientemente de cual de ambos padres fume durante la gestación se encuentra la asociación entre el tabaquismo gestacional y el desarrollo de la obesidad infantil.
La 11 beta deshidrogenasa de esteroide, es un agente regulador en el metabolismo de los glucocorticoides.

La relación pequeño al nacer demuestra la proporción en el desarrollo de diferentes enfermedades prevalentes y no solo a la obesidad central.

La teoría de Barker es el principal modelo explicativo para el desarrollo de múltiples enfermedades que se inician a edades tempranas, con posterior instauración en la adultez.

Se hallaron otros factores que predispone al desarrollo de la obesidad infantil. 


\section{CONTINUACIÓN TABLA I}

\begin{tabular}{ll}
\hline REFERENCIA & DEFINICION O RESULTADOS \\
& RECONCLUSIONES Y/O \\
\end{tabular}

25

28

37

41

\begin{abstract}
La nicotina causa una disminución de flujo sanguíneo placentario a causa directa de la nicotina con el aumento de la resistencia vascular placentaria y en consecuencia a una disminución del oxígeno.
\end{abstract}

Se analiza los 4000 componentes que constituían el humo del cigarrillo y sus efectos en ratas embarazadas y después del parto; se pudo dilucidar que la nicotina era el principal agente en el aumento de la grasa corporal de las ratas.

Utilizando la técnica BeadArray se vinculó el tabaquismo gestacional y con patrones de metilación placentarios alterados importantes en la respuesta al estrés, entre los cuales se encontró los genes NR3C1 y HSD11B2.

La OMS, afirma la relación entre la obesidad y los diferentes factores de riesgo para el desarrollo de enfermedades no transmisibles.
La nicotina es el agente responsable de las modificaciones del flujo sanguíneo placentario, y sus efectos con el peso del infante.

El humo de cigarrillo aumenta el peso corporal en los niños directamente expuestos al humo del cigarrillo.

La metilación aberrante post-exposición tabaco de los genes NR3C1 relacionados con el receptor HSD11B2 implicado en la respuesta al estrés posiblemente aumente el riesgo para el aumento de peso.

Diferentes factores de riesgo se ven presentados en el desarrollo de las enfermedades no transmisibles.

\section{Tabaquismo durante la gestación}

Alrededor del $30 \%$ de las mujeres dejan de fumar espontáneamente o reducen la cantidad de cigarrillos voluntariamente al saber que están embarazadas. Los efectos del tabaco durante la gestación pueden ocasionar desprendimiento de placenta, placenta previa, embarazo ectópico y aborto espontáneo $(11,12)$. Sin embargo, en España, la prevalencia de mujeres gestantes fumadoras es independientemente de la clase social y de la edad de las madres e incluso el tabaquismo en esta población alcanza porcentajes de 30 a 35\% $(13,14)$. Por su parte, en países desarrollados, la prevalencia de tabaquismo en las mujeres embarazadas varía con la edad, las mujeres mayores de 30 años fuman menos, que las mujeres de 15 a 24 años, y abandonan este hábito entre el $20-40 \%$ de las fumadoras (15).

\section{Efecto directo del tabaco en el peso del infante}

El último reporte de la Organización Mundial de la Salud (OMS) del 2015 describe un aumento considerable de la obesidad infantil desde el año
1990 hasta el 2013, pasando de un total de $32 \mathrm{mi}-$ llones de niños tanto lactantes como escolares a 42 millones obesos, un $25 \%$ más que en las décadas anteriores, superando en un $30 \%$ el rango de la prevalencia esperada, lo cual permite proyectar para el año 2025 un total de 70 millones de niños obesos si la tendencia sigue igual (16).

La OMS ha reportado que la prevalencia aumentada de enfermedades metabólicas como la diabetes y las enfermedades cardiovasculares han sido asociadas con la obesidad en edades tempranas (16), la cual podría depender de una predisposición genética, hábitos nutricionales inadecuados, baja actividad física, lo cual puede favorecer el almacenamiento de nutrientes en forma de tejido adiposo alrededor de todo el cuerpo (17). El tabaquismo durante la gestación puede causar principalmente restricción del crecimiento intrauterino (RCIU) y parto pretérmino (3), sin embargo, se ha determinado una asociación adicional con desordenes metabólicos incluyendo la obesidad y el sobrepeso a edades tempranas $(17,18)$. En Estados Unidos, 15 millones de niños desde neonatos hasta escolares se encuentran expuestos al humo del tabaco (13). 
En un estudio publicado por Mizutani y cols en 2007 (19), demostró una relación significativa entre el tabaquismo gestacional y la obesidad infantil en la descendencia, usando como población de estudio niños nacidos entre el 1 de enero de 1991 y el 31 de diciembre de 1999 en la ciudad de Enzan, Japón; del proyecto Enzan, un estudio de cohorte prospectivo en curso de las mujeres embarazadas y sus hijos que comenzó en 1988. De las maternas que dieron a luz a 1812 bebés, se logró obtener información de los estilos de vida materno de 1417 niños, y de esta última población, el 5,4\% fumaron durante la gestación. La prevalencia de sobrepeso infantil fue significativamente mayor entre los niños cuyas madres fumaron durante el embarazo que entre aquellos cuyas madres no lo hicieron, con un OR ajustado de 2,15 (IC 95\%: 1,12 - 4,11) e igualmente para obesidad con OR ajustado de 3,9 (IC 95\%: 1,46 - 10,56).

Posteriormente, en el 2009 Suzuki y cols (20), realizaron un nuevo análisis con nuevas valoraciones antropométricas a la población de estudio anterior del proyecto Enzan, ahora llamado proyecto Koshu. Se continuó observando una asociación entre el tabaquismo materno y el sobrepeso en los niños a los 9-10 años de edad con un OR ajustado de 1,9 (IC 95\%, 1,03-3,53) y para la obesidad un OR ajustado de 2,56 (IC 95\%: 1,02-6,38). Finalmente, en el año 2013 se decide realizar un nuevo análisis debido a las diferencias encontradas entre los OR ajustados para la asociación entre tabaquismo gestacional y el desarrollo de obesidad y sobrepeso en la misma población a los 5 años y los 9-10 años de edad; se usaron 2 cohortes: la $1^{\circ}$ cohorte formada por 1644 bebés con los cuales se hizo un seguimiento hasta los 5 años y, la $2^{\circ}$ corte formada por 1131 niños de la población anterior que tuvieron peso normal y que fueron seguidos hasta los 9 años. Se encontró una asociación significativa en los niños varones de la primera cohorte con un OR ajustado de 4,5 (IC 95\%: 2,2-10,2). Estos resultados sugieren que los efectos del tabaquismo materno durante el embarazo sobre el peso del infante tienden a manifestarse antes de los 5 años de edad, especialmente en niños varones (21).

\section{Tabaquismo no gestacional implicado en el de- sarrollo de la obesidad infantil}

En el año 2008, von Kries y cols (22), publicaron un estudio descriptivo realizado en Baviera, Alemania, con una población total de 5899 niños con una edad media de 5,8 años de edad, de los cuales 3067 eran niños y 2832 niñas; de éstos 2107 estuvieron expuesto al humo de cigarrillo por algún miembro de la familia y 3792 no estuvieron expuestos, encontrándose una prevalencia global de sobrepeso y obesidad de $19,2 \%$ y $7,1 \%$, respectivamente, en comparación con una menor prevalencia en los niños no expuestos al humo de cigarrillo de $10,9 \%$ y $2,6 \%$ para sobrepeso y obesidad, respectivamente. Teniendo en cuenta como posibles variables de confusión, grado de escolaridad de ambos padres, peso al nacer, IMC paternos, lactancia materna, actividad física y dedicación a videojuegos u otro material audiovisual, los resultados del estudio mostraron que los niños que crecieron en un ambiente extrauterino de padres fumadores, son más propensos al desarrollo de sobrepeso y obesidad infantil en comparación con aquellos que no fueron expuestos al humo del cigarrillo.

En el año 2014, Riedel y cols (23), realizaron una búsqueda en MEDLINE (1950 a diciembre 2013), EMBASE (1974 a diciembre de 2013) y Web of Knowledge (1900 a diciembre de 2013), para evidenciar si tanto el tabaquismo materno durante el embarazo y el tabaquismo paterno son factores predisponentes para desarrollar obesidad o sobrepeso en los niños. Los autores hallaron una asociación causal para el desarrollo de sobrepeso u obesidad en el niño expuesto al humo de cigarrillo tanto por la madre (OR agrupado de 1,33 para el sobrepeso y un OR 1,60 para la obesidad), como por el padre (OR agrupado 1,07 para el sobrepeso y OR agrupado 1,23), siendo la magnitud de asociación con el tabaquismo paterno inferior al de la madre.

\section{Tabaquismo materno como inductor de ambien- tes adversos intrauterinos}

El tabaquismo materno podría desencadenar el desarrollo de la obesidad infantil a través de diferentes mecanismos bioquímicos, moleculares y epigenéticos (24). En una revisión realizada en el año 2004 por Duran y cols $(5,6)$, se describe "la teoría de Barker", la cual ha sido presentada en forma dinámica como una teoría que podría explicar el desarrollo de múltiples enfermedades metabólicas y cardiovasculares en el niño y en el adulto; la teoría se basa en tres fases: (1) trastornos en la nutrición fetal que desencadenan cambios estructurales y funcionales en diferentes órganos y sistemas; (2) cambios bioquímicos y clínicos, que constituyen variables intermedias en la evolución de la enfermedad y, (3) la presencia de la enfermedad propiamente dicha. En el 2012 Wojtyla y cols (25), describen una disminución de flujo sanguíneo placentario a causa directa de la nicotina con el aumento de la resistencia vascular placentaria y en consecuencia a una disminución de la oxigenación fetal, debido a la vasoconstricción producida por la unión de la nicotina con receptores de acetilcolina ubicados en las arterias uterinas. Se supone, que lo anterior podría propiciar un medio adverso para el desarrollo fetal, lo cual conllevaría a una reprogramación genética para la adaptación a este medio, desarrollándose una absorción nutricional 
mejorada para un adecuado proceso metabólico; sin embargo, este cambio adaptativo podría tener repercusiones a mediano o largo plazo, cuando el ambiente adverso no sea un estado predominante sino por el contrario, abundante en nutrientes, generándose un almacenamiento excesivo de sustratos, que finalmente conduzcan a un estado de obesidad en los individuos con respecto a aquellos que no realizaron esta reprogramación $(26,27)$.

\section{Regulación epigenética de la obesidad mediada por el tabaquismo materno}

Se conocen 4 modos de regulación epigenética: 1.Regulación mediada por RNA no codificante, 2. Modificaciones de las histonas, 3. Modificaciones en la impronta, 4. Metilación del ADN, siendo esta ultima la más estudiada, que consiste en la adición de un grupo metilo a la posición $5^{\prime}$ de la citosina en el ADN, adoptando una conformación transcripcionalmente estable, reprimiendo o silenciando su expresión genética, particularmente cuando la metilación se produce dentro de las regiones promotoras de los genes (28); estas marcas de metilación se encuentran a menudo en grupos de pares de dinucleótidos de citosina-guanina llamados islas CpG (28). En presencia de la metilación del ADN, las proteínas que normalmente se unen a éste y permiten su transcripción son incapaces de unirse o en absoluto, limitan y detiene la transcripción (4). Los patrones de metilación normales son críticos para muchas funciones celulares, particularmente en la placenta, donde el funcionamiento celular correcto es crucial para el desarrollo fetal (28).

Hallazgos importantes utilizando la placenta humana han revelado asociaciones entre el tabaquismo gestacional y la metilación del ADN en genes específicos; por su parte, Suter y cols (29), observaron una asociación entre el consumo de tabaco materno y la metilación aberrante del ADN placentario e igualmente sugieren que el tabaquismo materno durante el embarazo se asocia con una alteración en la metilación de CpG específicos que se correlaciona con cambios importantes de la expresión génica en vías cruciales para asegurar el crecimiento y el desarrollo adecuado. En un estudio publicado en el 2015 (28), se determinó a través de la técnica BeadArray, que el tabaquismo materno podía inducir la alteración de algunos patrones de metilación de genes placentarios como NR3C1, CYP1A1, HTR2A, y HSD11B. NR3C1, conocido como el gen del receptor de glucocorticoides y HSD11B2 como el gen de la deshidrogenasa$11 \beta$-hidroxiesteroide tipo 2 (11- $\beta$-HSD2), tienen un papel importante en la respuesta al estrés. El estado de metilación de NR3C1 en la placenta se ha asociado previamente con el peso infantil al nacimiento y con el neurocomportamiento, y el estado de metilación del gen 11- $\beta$-HSD placentario se ha asociado con el crecimiento infantil y también con el neurocomportamiento. Por su parte, la enzima $11-\beta-H S D 2$ cataliza la conversión de cortisol activo en cortisona inactiva, regulando así la disponibilidad de los glucocorticoides a su receptor. El cortisol placentario también se asocia con el aumento de peso posnatal, reiterando, de esta manera, el potencial de esta vía como un posible parámetro indicativo del estado de salud de los niños (28).

\section{DISCUSIÓN}

Algunos estudios demuestran que una de cada cinco mujeres embarazadas en países desarrollados fuman y la declaración de la materna en cuanto al hábito de fumar durante su gestación, se ha considerado la prueba más valiosa y sencilla para confirmar esta acción, dado que no tiene ningún costo; sin embargo, algunas gestantes tienden a ocultar esta condición y es cuando se procede a ayudas diagnósticas como la medición de monóxido de carbono en el aire espirado y la cotinina en saliva $u$ orina (30-33). Por su parte, la cotinina puede considerarse uno de los marcadores más importantes de detección, dada su alta sensibilidad para demostrar consumo bajo o esporádico de tabaco, pero el principal inconveniente para la utilización de esta prueba diagnóstica es su poca accesibilidad y alto costo (34). La medición de monóxido de carbono en aire espirado tiene limitaciones, ya que la vida media del monóxido de carbono es corta, entre 1 y 4 horas, lo cual posibilita la generación de varios falsos negativos $(35,36)$.

El tabaquismo materno durante la gestación ha sido considerado como un factor de riesgo para el RCIU y parto pretérmino (3). Sin embargo, ha cobrado importancia en cuanto a sus posibles implicaciones sobre el resultado gestacional y la salud del infante; específicamente, se ha relacionado con el bajo peso al nacer y con un mayor riesgo de desarrollar obesidad infantil en la descendencia $(19,20,37)$.

En un estudio realizado por Wojcicki y Heyman (38), se describe la existencia de múltiples factores implicados en el desarrollo del sobrepeso y la obesidad en el niño, que incluyen el consumo de cigarrillo durante la gestación y la lactancia materna. El mecanismo implicado es una alteración de la regulación a largo plazo en el balance energético que puede tener efectos deletéreos sobre procesos epigenéticos y probablemente sobre el desarrollo de los circuitos hipotalámicos que equilibran el peso, la función pancreática endocrina, la proporción fenotípica del binomio musculo-adiposidad, y otros ciclos de reprogramación metabólicos del cuerpo. Por su parte, Delisle y cols (39), han abordado la relación que existe entre el peso al momento del nacimiento y el desarrollo posterior de enfermedades prevalentes en la adultez, como la obesidad; es 
decir, que los autores no sólo hacen referencia a la obesidad en el niño sino a la obesidad que podría persistir incluso en la edad adulta. En este estudio no se demostró una relación causal, ya que las alteraciones en la programación fetal pueden ser el resultado de un conjunto de factores adicionales como la pobreza, el sedentarismo, la dieta "aterogenica", y la predisposición genética, que conllevan posiblemente a una programación alterada y posterior bajo peso al nacer y enfermedad.

Aunque los hallazgos de los anteriores estudios son concluyentes, no se realizó un adecuado control de otras variables confusionales como, situación socioeconómica, nivel educativo de los padres, estado nutricional de la madre, farmacocinética, farmacodinamia y metabolismo nicotínico o el metabolismo de los demás 4000 xenobióticos que se encuentran en el humo del cigarrillo $(4,40,41)$. En el 2008, von Kries (22), lidera un estudio que pretendía esclarecer el efecto de algunas variables de confusión en cuanto el efecto del tabaquismo gestacional sobre la salud del niño, como el consumo de cigarrillo por el cónyuge o por otros convivientes de la madre, y el consumo de cigarrillo antes o después de la gestación. Se demostró que la asociación entre el tabaquismo gestacional y la obesidad infantil no se limitaba al tabaquismo materno, ya que el paterno explicaba parcialmente esta asociación.

\section{CONCLUSIÓN}

La exposición al humo del cigarrillo durante la gestación se relaciona con alteraciones en el crecimiento del niño y en el desarrollo de enfermedades prevalentes asociadas a la obesidad. Se sugiere la realización de más estudios donde se lleve a cabo un mayor control de algunas variables de confusión $y$, realizar campañas en contra de la obesidad infantil y promover la disminución del consumo del tabaco en los países en vía de desarrollo, para reducir la aparición de posibles enfermedades no transmisibles en la adultez.

Agradecimientos: Los autores agradecen la asesoría y acompañamiento temático del doctor Edgar Noreña, docente y médico gineco-obstetra de la Facultad de Ciencias de la Salud de la Corporación Universitaria Remington. A.M.G.V fue becaria de estudios doctorales del Departamento Administrativo de Ciencia, Tecnología e Innovación de Colombia.

\section{REFERENCIAS}

1. Informe OMS sobre la epidemia mundial de tabaquismo [Internet]. 2008. Available from: http://apps.who. int/iris/bitstream/10665/43897/1/9789243596280_ spa.pdf.

2. Tong VT, Dietz PM, Morrow B, D'Angelo DV, Farr SL, Rockhill KM, et al. Trends in smoking before, during, and after pregnancy-Pregnancy Risk Assessment Monitoring System, United States, 40 sites, 20002010. MMWR Surveill Summ. 2013;62(6):1-19.

3. Maggiolo J. Tabaquismo durante el embarazo. Rev Neumol Pediatr. 2011;6(1):12-5.

4. Knopik VS, Maccani MA, Francazio S, McGeary JE. The epigenetics of maternal cigarette smoking during pregnancy and effects on child development. Dev Psychopathol. 2012;24(4):1377-90.

5. Barker DJP. Mothers, babies, and health in later life: Elsevier Health Sciences; 1998.

6. Durán P. Nutrición temprana y enfermedades en la edad adulta: acerca de la" hipótesis de Barker". Arch Argentinos Pediatr. 2004;102(1):26-34.

7. Chan M. Global status report on noncommunicable diseases. OMS. 2010;1(1):17-8. Disponible en: http://www.who.int/nmh/publications/ncd_report_full_ en.pdf.

8. WHO. Cause-specific mortality, 2008: WHO region by country. Geneva: WHO. 2011. Disponible en: http:// apps.who.int/searo-rho/node.main.887?lang=en.

9. Murray CJ, Vos T, Lozano R, Naghavi M, Flaxman AD, Michaud C, et al. Disability-adjusted life years (DALYs) for 291 diseases and injuries in 21 regions, 19902010: a systematic analysis for the Global Burden of Disease Study 2010. Lancet. 2013;380(9859):2197223.

10. Bloom DE, Cafiero E, Jané-Llopis E, Abrahams-Gessel S, Bloom LR, Fathima S, et al. The global economic burden of noncommunicable diseases. Program on the Global Demography of Aging, 2012.

11. Cnattingius S, Mills JL, Yuen J, Eriksson O, Salonen $H$. The paradoxical effect of smoking in preeclamptic pregnancies: smoking reduces the incidence but increases the rates of perinatal mortality, abruptio placentae, and intrauterine growth restriction. Am J Obstet Gynecol. 1997;177(1):156-61.

12. Wright $A$, Walker J. Drugs of abuse in pregnancy. Best Pract Res Clin Obstet Gynaecol. 2001;15(6):987-98.

13. Gilliland FD, Berhane K, McConnell R, Gauderman WJ, Vora H, Rappaport EB, et al. Maternal smoking during pregnancy, environmental tobacco smoke exposure and childhood lung function. Thorax. 2000;55(4):271-6.

14. Pichini S, Puig C, García-Algar Ó, Pacifici R, Figueroa $\mathrm{C}$, Vall $\mathrm{O}$, et al. Efectos neonatales del hábito tabáquico durante el embarazo y determinantes sociodemográficos en Barcelona. Med Clín. 2002;118(2):53-6.

15. Rantakallio P. Relationship of maternal smoking to morbidity and mortality of the child up to the age of five. Acta Paediatrica. 1978;67(5):621-31.

16. OMS. Datos y cifras sobre obesidad infantil. http:// wwwwhoint/end-childhood-obesity/facts/es/. 2015.

17. Mahmood SS, Levy D, Vasan RS, Wang TJ. The Framingham Heart Study and the epidemiology of cardiovascular disease: a historical perspective. Lancet. 2014;383(9921):999-1008.

18. Behl M, Rao D, Aagaard K, Davidson TL, Levin ED, Slotkin TA, et al. Evaluation of the association between maternal smoking, childhood obesity, and metabolic disorders: a national toxicology program workshop review. Environ Health Perspect. 2012;121(2):170-80.

19. Mizutani T, Suzuki K, Kondo N, Yamagata Z. Association of maternal lifestyles including smoking during pregnancy with childhood obesity. Obesity. 2007;15(12):3133-9. 
20. Suzuki K, Ando D, Sato M, Tanaka T, Kondo N, Yamagata $Z$. The association between maternal smoking during pregnancy and childhood obesity persists to the age of 9-10 years. J Epidemiol. 2009;19(3):13642.

21. Suzuki K, Sato M, Ando D, Kondo N, Yamagata Z. Differences in the effect of maternal smoking during pregnancy for childhood overweight before and after 5 years of age. J Obstet Gynaecol Res. 2013;39(5):91421.

22. von Kries R, Bolte G, Baghi L, Toschke AM. Parental smoking and childhood obesity-is maternal smoking in pregnancy the critical exposure? Int $\mathrm{J}$ Epidemiol. 2008;37(1):210-6.

23. Riedel C, Schönberger K, Yang S, Koshy G, Chen Y-C, Gopinath B, et al. Parental smoking and childhood obesity: higher effect estimates for maternal smoking in pregnancy compared with paternal smoking-a meta-analysis. Int J Epidemiol. 2014;43(5):1593-606.

24. Lucas A. Programming by early nutrition in man. The childhood environment and adult disease. Ciba Foundation Symposium;1991:38-55.

25. Wojtyla A, Gozdziewska M, Paprzycki P, Bilinski P. Tobacco-related foetal origin of adult diseases hypothesis-population studies in Poland. Ann Agric Environ Med. 2012;19(1).

26. Knopik VS, Maccani MA, Francazio S, McGeary JE. The epigenetics of maternal cigarette smoking during pregnancy and effects on child development. Dev Psychopathol. 2012;24(04):1377-90.

27. Oken E, Gillman MW. Fetal origins of obesity. Obes Res. 2003;11(4):496-506.

28. Maccani JZ, Maccani MA. Altered placental DNA methylation patterns associated with maternal smoking: current perspectives. Adv Genomics Genet. 2015;2015(5):205-14.

29. Suter M, Ma J, Harris AS, Patterson L, Brown KA, Shope $C$, et al. Maternal tobacco use modestly alters correlated epigenome-wide placental DNA methylation and gene expression. Epigenetics. 2011;6(11):128494.
30. Warren CW, Asma S, Lee J, Lea V, Mackay J. Global tobacco surveillance system. The GTSS Atlas Atlanta: CDC Foundation. 2009.

31. Pardell H, i Alentà HP, Saltó E, Salleras L. Manual de diagnóstico y tratamiento del tabaquismo: Ed. Médica Panamericana; 1996.

32. Aranda Regules JM, Mateos Vilchez P, González Villalba A, Sanchez F, Luna del Castillo JdD. Validez de distintas medidas de consumo de tabaco durante el embarazo: especificidad, sensibilidad y puntos de corte dónde y cuándo. Rev Esp Salud Pública. 2008;82(5):535-45.

33. Becoña E. Tratamiento psicológico del tabaquismo. Monografía Tabaco Adicciones. 2004;16(2):237-63.

34. Haley NJ, Sepkovic DW, Hoffmann D. Elimination of cotinine from body fluids: disposition in smokers and nonsmokers. Am J Public Health. 1989;79(8):1046-8.

35. Frederiksen LW, Martin JE. Carbon monoxide and smoking behavior. Addict Behav. 1979;4(1):21-30.

36. Jarvis M, Russell M, Saloojee $Y$. Expired air carbon monoxide: a simple breath test of tobacco smoke intake. Br Med J. 1980;281(6238):484.

37. Hunter DJ, Reddy KS. Noncommunicable diseases. N Engl J Med. 2013;369(14):1336-43.

38. Wojcicki JM, Heyman MB. Let's move-childhood obesity prevention from pregnancy and infancy onward. N Engl J Med. 2010;362(16):1457-9.

39. Delisle H. La programmation fœtale des maladies chroniques liées à la nutrition. Cahiers d'études et de recherches francophones/Santé. 2002;12(1):56-63.

40. Lingas RI, Matthews SG. A short period of maternal nutrient restriction in late gestation modifies pituitaryadrenal function in adult guinea pig offspring. Neuroendocrinology. 2001;73(5):302-11.

41. Harris HR, Willett WC, Michels KB. Parental smoking during pregnancy and risk of overweight and obesity in the daughter. Int J Obes (London). 2013;37(10):135663. 